\section{The AM-GM Inequality and CBS Inequality Are Equivalent}

\section{Minghua LiN}

T his note is partly motivated by a recent one having the same nature [1], giving a short proof of the equivalence of the AM-GM (i.e., arithmetic-mean/geometricmean) inequality and the Bernoulli inequality. The present note provides a very short proof of equivalence of the AM-GM inequality and the CBS (i.e., Cauchy-BunyakovskySchwarz) inequality.

AM-GM Inequality If $c_{1}, \cdots, c_{n}$ are nonnegative real numbers, then

$$
\frac{1}{n} \sum_{k=1}^{n} c_{k} \geq \prod_{k=1}^{n} c_{k}^{\frac{1}{n}}
$$

CBS Inequality If $a_{1}, \cdots, a_{n}, b_{1}, \cdots, b_{n}$ are nonnegative real numbers, then

$$
\sum_{k=1}^{n} a_{k} \sum_{k=1}^{n} b_{k} \geq\left(\sum_{k=1}^{n} \sqrt{a_{k} b_{k}}\right)^{2} .
$$

(In both statements we clearly can assume without loss of generality that the scalars considered are positive.)

It may be quibbled that any two true statements are equivalent, so knowing these two inequalities suffices to prove their equivalence. Still it is striking that they can be proved one from the other so directly, as I will show.

To show (0.1) implies (0.2), we only need a special case of $(0.1)$

$$
\frac{c_{1}+c_{2}}{2} \geq \sqrt{c_{1} c_{2}} .
$$

Substituting into (0.3), we have

$$
\frac{a_{i} \sum_{k=1}^{n} b_{k}+b_{i} \sum_{k=1}^{n} a_{k}}{2} \geq \sqrt{a_{i} b_{i}} \sqrt{\sum_{k=1}^{n} a_{k} \sum_{k=1}^{n} b_{k}}
$$

for $i=1, \cdots, n$. Summing over $i$, we obtain

$$
\sum_{k=1}^{n} a_{k} \sum_{k=1}^{n} b_{k} \geq\left(\sum_{i=1}^{n} \sqrt{a_{i} b_{i}}\right) \sqrt{\sum_{k=1}^{n} a_{k} \sum_{k=1}^{n} b_{k}} .
$$

Thus (0.2) follows.

To show the converse, letting $b_{k}=\frac{1}{n^{2}}$ in (0.2) for all $k$, we have

$$
\frac{1}{n} \sum_{k=1}^{n} a_{k} \geq\left(\frac{1}{n} \sum_{k=1}^{n} \sqrt{a_{k}}\right)^{2} .
$$

Repeatedly using (0.4), we obtain

$$
\begin{aligned}
& \frac{1}{n} \sum_{k=1}^{n} a_{k} \geq\left(\frac{1}{n} \sum_{k=1}^{n} \sqrt{a_{k}}\right)^{2} \\
& \quad \geq\left(\frac{1}{n} \sum_{k=1}^{n} a_{k}^{\frac{1}{4}}\right)^{4} \geq \cdots \geq\left(\frac{1}{n} \sum_{k=1}^{n} a_{k}^{\frac{1}{2^{m}}}\right)^{2^{m}} \geq \cdots
\end{aligned}
$$

By L'Hospital's rule, it is easy to see that

$$
\lim _{x \rightarrow 0+} \ln \left(\frac{1}{n} \sum_{k=1}^{n} a_{k}^{x}\right) / x=\frac{1}{n} \sum_{k=1}^{n} \ln a_{k},
$$

so

$$
\lim _{x \rightarrow 0+}\left(\frac{1}{n} \sum_{k=1}^{n} a_{k}^{x}\right)^{\frac{1}{x}}=\prod_{k=1}^{n} a_{k}^{\frac{1}{n}} .
$$

Thus in (0.5), we can pass to the limit $m \rightarrow \infty$, giving $\frac{1}{n} \sum_{k=1}^{n} a_{k} \geq \prod_{k=1}^{n} a_{k}^{\frac{1}{n}} ;$ hence (0.2) implies (0.1).

\section{REFERENCE}

1. L. Maligranda, The AM-GM inequality is equivalent to the Bernoulli inequality, Math. Intelligencer, 34, no.1 (2012), 1-2.

Department of Applied Mathematics

University of Waterloo

Waterloo, Ontario N2L 3G1

Canada

e-mail: mlin87@ymail.com 